\title{
Bilateral Superior Cerebellar Artery Embolic Occlusion with a Fetal-Type Posterior Cerebral Artery Providing Collateral Circulation
}

\author{
Taylor J. Bergman Rachael C. Saporito Thomas Hope \\ Division of Neurology, Department of Internal Medicine, Mercer University School of \\ Medicine, Macon, GA, USA
}

\section{Keywords}

Superior cerebellar artery - Embolic occlusion - Fetal-type posterior cerebral artery · Collateral circulation · Cardioembolic stroke

\begin{abstract}
Bilateral infarction of the superior cerebellar arteries with sparing of the rest of the posterior circulation, particularly the posterior cerebral arteries, is an uncommon finding in neurological practice. Most commonly, the deficits of the superior cerebellar arteries and posterior cerebral arteries occur together due to the close proximity of their origins at the top of the basilar artery. A patient was transferred to the neurological intensive care unit with a history of recent-onset falls from standing, profound hypertension, dizziness, and headaches. The neurological exam revealed cerebellar signs, including dysmetria of the right upper extremity and a decreased level of consciousness. Computed tomography of the head and neck revealed decreased attenuation throughout most of the cerebellar hemispheres suggestive of ischemic injury with sparing of the rest of the brain. Further investigation with a computed tomography angiogram revealed a fetal-type posterior cerebral artery on the right side that was providing collateral circulation to the posterior brain. Due to this embryological anomaly, the patient was spared significant morbidity and mortality that would have likely occurred had the circulation been more typical of an adult male.

(C) 2016 The Author(s) Published by S. Karger AG, Basel
\end{abstract}

KARGER
Taylor Bergman

Medical Center of Central Georgia, West Tower

Box 30, 777 Hemlock Street

Macon, GA 31217 (USA)

E-Mail bergman_tj@med.mercer.edu 


\section{Case Reports in Neurology}

\section{Introduction}

Bilateral infarction of the superior cerebellar arteries (SCAs) with sparing of the rest of the posterior circulation, particularly the posterior cerebral arteries, is an uncommon finding in neurological practice. Typically, an embolism large enough to occlude both SCAs at the top of the basilar artery would also infarct the posterior cerebral vasculature. This case presents a departure from the presentation of a traditional posterior circulation cerebral infarction most likely as a result of a rare vascular anomaly rooted in an aberrant embryological process.

\section{Case Report}

A 63-year-old African American man was transferred to the neurological intensive care unit from a nearby hospital after presenting with an acute history of multiple falls. Earlier that day, he had fallen once while walking to the bathroom from his bed and had fallen again shortly thereafter while walking through his living room. The patient's family decided at that time to bring him to the nearby hospital for treatment. At that hospital, no focal findings were discovered, but his blood pressure was noted to be elevated at 272/139 $\mathrm{mm} \mathrm{Hg}$. At that point, the original hospital placed the patient on a nicardipine drip to treat his hypertension and transferred the patient to our medical center, where he arrived late that night.

The patient presented to the medical center complaining of dizziness and agonizing headache. He was also noted to be somnolent and experiencing some dysarthria. The family at the bedside reported that the patient's past medical history was significant for hypertension, diabetes mellitus type II, and hyperlipidemia. It was also determined that the patient was a former chronic smoker, did not consume alcohol, and used marijuana occasionally. Physical exam showed some lethargy, dysarthria, right gaze paresis and nystagmus, vertical gaze weakness, and dysmetria on the right side more so than on the left. There was no hemiparesis, and visual fields were normal to confrontation. The patient had 5/5 strength, intact sensation, and 2+ reflexes of the upper and lower extremities bilaterally. It was determined that the patient had 4 points on both the NIH Stroke Scale and the Modified Rankin Scale for Neurologic Disability at admission. Computed tomography (CT) of the head and neck revealed decreased attenuation throughout most of the cerebellar hemispheres suggestive of ischemic injury (Fig. 1), diminished caliber of the fourth ventricle indicating increased pressure, and an old watershed infarct on the right side between the territories of the middle cerebral artery and the posterior cerebral artery (PCA). The area of the brain that is perfused by the PCA was surprisingly completely spared from infarct. CT angiography of the head revealed diffuse abnormally decreased attenuation of the cerebellar hemispheres with sparing of the vermis, suggesting infarction of both SCAs, and a narrow irregular left vertebral artery with a risk for subtotal thrombosis. There was no evidence of stenosis or a blood clot in the basilar artery or either SCA at the time of the CT angiography. Furthermore, it was discovered that the patient had a partial fetal-type PCA (FPCA) originating from the internal carotid artery (ICA) on the right side as seen in Figure 2.

Due to the fact that the patient was well out of the window for thrombolytic therapy, he was initially treated with aspirin. Neurosurgery was consulted because of the patient's declining mental status caused by a mass effect in the posterior fossa. They elected to immediately take the patient in for a decompressive craniotomy. During the procedure, the dura overlying the cerebellum was noted to be very taut and to have a blue discoloration. Upon 


\section{Case Reports in Neurology}

incision of the dura, there was spontaneous protrusion of necrotic brain matter. Much of the left and right cerebellum hemispheres were necrotic, but the vermis appeared to be spared from damage. The necrotic tissue was cleared, and the patient's cranium was closed.

Over the next $24 \mathrm{~h}$ after the operation, the patient was extubated and remained lethargic but arousable. He responded to verbal commands, and facial movements were symmetrical. His right lateral gaze weakness persisted, and movement was diminished somewhat in the left extremities. Additionally, CT of the head showed new hydrocephalus, and electrocardiogram monitoring revealed intermittent atrial fibrillation throughout the day. Transthoracic echocardiogram was performed after discovery of the patient's arrhythmia, and it showed an ejection fraction of 55-60\% as well as no evidence of residual thrombus. An amiodarone drip was administered in an effort to normalize the patient's heart rhythm. Anticoagulation with warfarin was not prescribed for another 3 weeks, as the patient experienced some bleeding in the posterior fossa after surgery. Neurosurgery placed an external ventricular drain to help decrease the intracranial pressure.

Prior to the discovery of this patient's atrial fibrillation, the intensive care team had proposed that the cause of the stroke might have been an embolism from the diseased left vertebral artery. However, knowing that this patient was experiencing intermittent atrial fibrillation led the team to assess that the cause was more likely an embolism from the left atrium. Because it is unlikely that 2 separate emboli occluded both SCAs simultaneously, it was postulated that this was a single top of the basilar artery embolism that was large enough to occlude both SCAs. Such an occlusion should have led to ischemic injury of the regions of the brain perfused by the PCAs. Given this, the team determined that the bilateral PCA sparing may have been due to collateral perfusion supplied by the fetal origin of the PCA on the right side.

The patient remained lethargic over the next $24 \mathrm{~h}$ despite the placement of the external ventricular drain. The next morning, however, the patient woke up with markedly improved symptomatology. He was oriented to person and place, followed commands appropriately, and had 5/5 strength in all extremities along with intact sensation. Dysarthria and dysmetria of both upper extremities were noted, but overall the patient made a remarkable recovery from his condition the day before. The team continued to monitor and treat the patient over the next 3 weeks, and the patient was discharged for rehabilitation. At discharge, it was determined that the patient once again had 4 points on both the NIH Stroke Scale and the Modified Rankin Scale for Neurologic Disability.

\section{Discussion}

On review of the literature, this may be the first reported case in which an fPCA provided collateral circulation to the posterior brain after an embolus had occluded the top of the basilar artery. As stated earlier, this was almost certainly a single embolism occluding the top of the basilar artery rather than 2 separate emboli occluding both SCAs simultaneously. The embolus itself is presumed to have been cardiac in origin given the incidental finding of intermittent atrial fibrillation on this patient's electrocardiogram monitoring during his admission. An embolus large enough to occlude both the left and right SCAs would presumably obstruct arterial blood flow beyond the top of the basilar artery. Thus, an event such as this without a source of collateral circulation would lead to infarction of the areas of the brain perfused by the PCA. Patients that experience bilateral PCA occlusion quickly develop visual defects as infarction of the occipital lobe develops. The most severe manifestation one would 
experience in an event such as this is cortical blindness [1]. This patient's visual fields remained intact throughout this entire case, further indicating that both of his PCAs were spared from ischemia.

In a patient with an PPCA, the PCA originates from and primarily depends on circulation from the ICA rather than from the basilar artery. During embryonic development, the ICA anastomoses with the developing neural arteries to form the posterior communicating arteries (PCOM). There are 3 carotid-to-basilar anastomoses that form during development, but only the PCOM persists into adulthood [2]. The PCAs thus originate as extensions of the PCOMs in normal development. Following this, the vertebrobasilar arterial system develops and anastomoses to the bilateral PCAs. Initially, the arterial segment between the basilar artery and the PCOM, referred to as the P1 segment, is smaller in caliber than the PCOM itself. As development proceeds, the caliber of the PCOM regresses while the P1 segment simultaneously increases in caliber. Thus, in the adult configuration of cerebral vasculature, the P1 segment is larger in diameter than the PCOM, and the vertebrobasilar system provides the majority of the posterior blood flow [3].

During embryonic development, if a patient's P1 segment remains hypoplastic or is completely absent, the patient has an fPCA. In situations where the patient's P1 segment is completely absent, this is defined as a complete fPCA. Patients with a hypoplastic P1 segment are said to have a partial fPCA [3]. A CT angiogram or magnetic resonance imaging of the head helps differentiate between these 2 definitions. These fPCAs can be unilateral or bilateral. Our patient was determined to have a unilateral, right-sided partial fPCA based on his CT angiogram (Fig. 2). The prevalence of fPCAs has varied widely in the literature since the developmental anomaly was first discovered by autopsy and more recently by radiographic imaging. For unilateral partial fPCAs, like the one seen in this case, the prevalence has ranged from 11 to $29 \%$ with a wide range of study populations from healthy individuals to subjects with neurological dysfunction or infarction [3].

The patient's symptoms are more consistent with SCA involvement along with some possible rostral brainstem infarction. The most prominent symptoms seen in SCA infarction are dysarthria and ataxia involving both the extremities and the trunk, which were both seen in this patient's presentation. Associated nausea, dizziness, and headache are also commonly seen [4]. Vertical gaze palsy and horizontal gaze weakness are both clinical signs exhibited by this patient that indicate that he experienced rostral brainstem infarction [1, 5]. His somnolence throughout his clinical course is another symptom that may have resulted from ischemia of the brainstem [1].

The prognosis of basilar artery occlusion is generally poor. In a study conducted by Devuyst et al. [6], 54\% of patients with basilar artery occlusion or basilar artery stenosis of greater than $50 \%$ suffered from mortality or severe disability, while $34 \%$ experienced moderate disability. Additionally, cardioembolic cerebral infarctions carry a higher in-hospital mortality in the acute setting than any other type of ischemic stroke. Prognoses in these cases are also worsened by the fact that cardioembolic strokes are associated with a higher rate of functional impairment at discharge [7]. Collateral circulation is vital for sustaining an adequate amount of perfusion to the brain in circumstances where 1 or multiple cerebral arteries are obstructed. In this case, a developmental anomaly led to the prevention of anoxic brain injury through collateral circulation. A unilateral fPCA may have reduced both the short-term and long-term morbidity and mortality of an embolic occlusion of the basilar artery. 


\section{Conclusion}

The symptomatology of the patient did not align with all of the deficits that usually present with an infarction of the posterior circulation, and the radiological workup revealed an fPCA on the right side. The case illustrates a rare clinical circumstance in which a developmental cerebrovascular anomaly provided collateral circulation that likely reduced the morbidity and potentially the mortality of an ischemic stroke by minimizing the amount of infarcted brain matter. Studies centered on fPCAs typically focus on whether these anomalies are associated with an increased incidence of stroke, but this case presents a unique situation in which it instead helped alleviate the consequences of a stroke.

\section{Statement of Ethics}

The patient provided informed consent for the publication of this case report.

\section{Disclosure Statement}

The authors have nothing to disclose. This case report was not funded.

\section{References}

1 Caplan LR: "Top of the basilar" syndrome. Neurology 1980;30:72-79.

2 Kathuria S, Gregg L, Chen J, Gandhi D: Normal cerebral arterial development and variations. Semin Ultrasound CT MRI 2011;32:242-251.

-3 van Raamt AF, Mali W, van Laar PJ, van der Graaf Y: The fetal variant of the circle of Willis and its influence on the cerebral collateral circulation. Cerebrovasc Dis 2006;22:217-224.

4 Terao SI, Sobue G, Izumi M, Miura N, Takeda A, Mitsuma T: Infarction of superior cerebellar artery presenting as cerebellar symptoms. Stroke 1996;27:1679-1681.

5 Ferbert A, Bruckmann H, Drummen R: Clinical features of proven basilar artery occlusion. Stroke 1990;21:1135-1142.

6 Devuyst G, Bogousslavsky J, Meuli R, Moncayo J, de Freitas G, van Melle G: Stroke or transient ischemic attacks with basilar artery stenosis or occlusion: clinical patterns and outcome. Arch Neurol 2002;59:567-573.

7 Arboix A, Alio J: Acute cardioembolic cerebral infarction: answers to clinical questions. Curr Cardiol Rev 2012;8:54-67. 


\section{Case Reports in Neurology}

\begin{tabular}{l|l}
\hline Case Rep Neurol 2016;8:258-263 \\
\hline DOI: 10.1159/000452942 & $\begin{array}{l}\text { C 2016 The Author(s). Published by S. Karger AG, Basel } \\
\text { www.karger.com/crn }\end{array}$ \\
\hline
\end{tabular}

Bergman et al.: Bilateral Superior Cerebellar Artery Embolic Occlusion with a Fetal-Type Posterior Cerebral Artery Providing Collateral Circulation

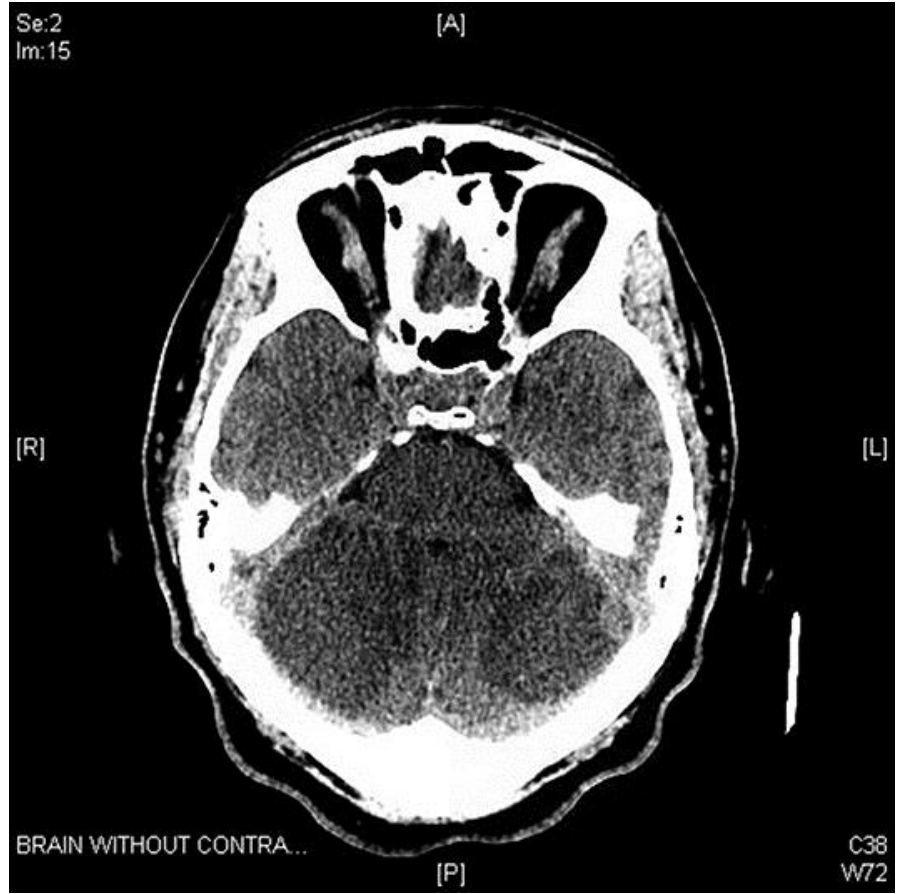

Fig. 1. Decreased attenuation of both cerebellar hemispheres.

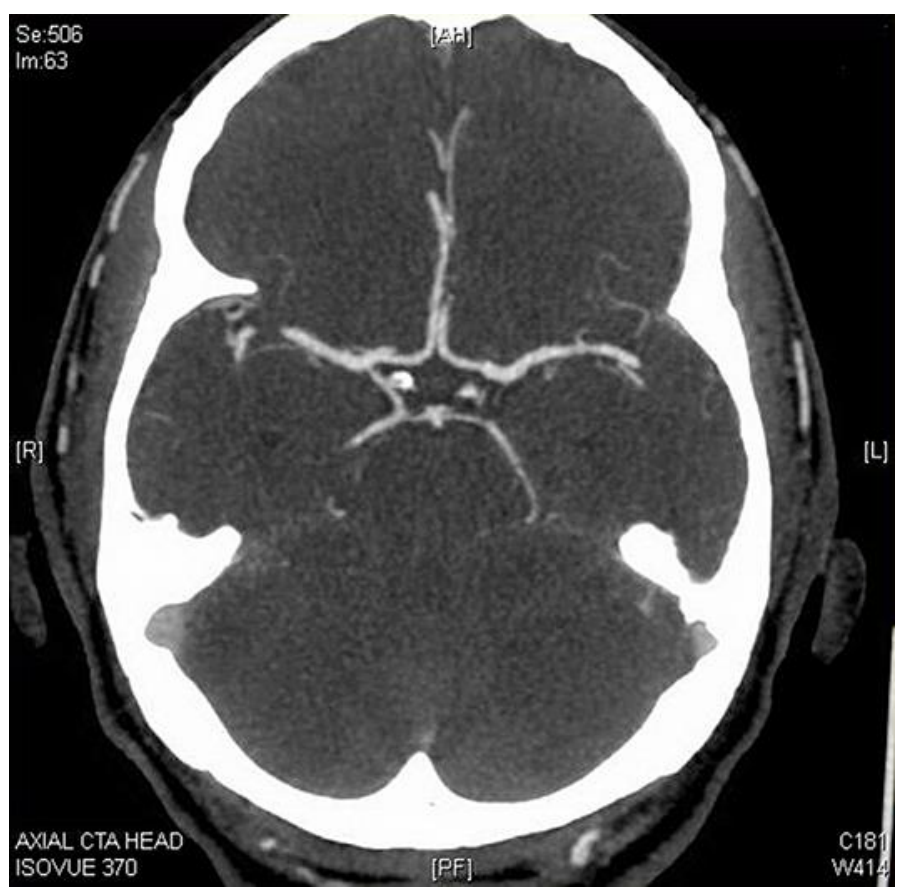

Fig. 2. Right-sided fetal-type posterior cerebral artery. 